\title{
Automated Registration and Fusion of Functional and Anatomical MRI for Navigated Neurosurgery
}

\author{
T. Rohlfing ${ }^{1}$, J. Beier ${ }^{1}$, J. B. West ${ }^{2}$, \\ U.-W. Thomale ${ }^{3}$, T. Liebig ${ }^{1}$, and C. A. Taschner ${ }^{1}$ \\ 1 Department of Radiology, Charité, Campus Virchow-Hospital \\ Medical Faculty of Humboldt-University Berlin \\ Augustenburger Platz 1, D-13353 Berlin, Germany \\ \{torsten.rohlfing, juergen. beier\}@charite.de \\ $\{$ christian.taschner, thomas.liebig\}@charite.de \\ 2 Department of Computer Science \\ Vanderbilt University, Nashville, TN, USA \\ jayw@vuse .vanderbilt.edu \\ 3 Department of Neurosurgery, Charité, Campus Virchow-Hospital \\ Medical Faculty of Humboldt-University Berlin \\ Augustenburger Platz 1, D-13353 Berlin, Germany \\ uthomale@charite.de
}

\begin{abstract}
A procedure for acquisition, automated registration and fusion of functional and anatomical magnetic resonance images (MRI) is presented and validated. The technique is based upon the acquisition of high-resolution anatomical slices at the same spatial locations as functional images ( 5 slices). The accuracy of registration of these slices and high-resolution 3D MRI volumes (MP-RAGE imaging) was quantified using adapted data originating from the Vanderbilt retrospective registration project (8 patients). Selecting a subset of slices from that data, the small number of images available from fMRI acquisition was taken into account. Quantitative analysis showed no loss of accuracy caused by the reduced number of slices used for registration. For real patient data, fMRI were fused with MP-RAGE images, thus integrating anatomical images with information about locations of functional areas. Via a case study, the benefits of the described approach for intraoperative navigation using an operating microscope (MKM, Zeiss) are demonstrated.
\end{abstract}

\section{Introduction}

Intraoperative navigation in neurosurgery requires knowledge of the exact positions of lesions. In addition, information about the locations of certain functions areas in the operating region helps to protect these risk structures from accidental damage.

Positron emission tomography (PET) and single photon emission tomography (SPECT) deliver suitable information by displaying the spatial distribution 
of radionuclides inside the patient's body. These nuclides are attached to substances like glucose, and their concentration provides information about local metabolic activity.

An alternative to these techniques is based upon the so-called "BOLD effect" (Blood Oxygenation Level Dependency). MRI gradient echo sequences (e.g. T2*-weighted) are sensitive to blood oxygenation. Exploiting this dependency, functional MRI (fMRI, cf. [1]) is computed from a sequence of plain MRI images. In order to do this, the pixel intensities of the images are correlated with excitations of certain areas of interest.

While PET and SPECT are primarily useful for locating brain lesions, fMRI identifies healthy functional areas of the brain. Using this information, therapy can be planned in such a way as to interfere with these areas as little as possible. For these reasons it is desirable to integrate fMRI rather than PET into the process of planning neurosurgical interventions. Even more benefit is to be expected if fMRI data is also available for navigation during surgery, thus enabling the surgeon to assess the locations of access paths and resections in relation to certain functional regions to be protected [2].

Another advantage of fMRI becomes recognizable as soon as fusion with other modalities is performed, in particular those that display anatomical information. Integrated into the acquisition process, high resolution anatomical slices are collected at the same locations as the fMRI slices.

Voxel-based automatic registration algorithms can then be employed for robust correlation of these slices with another anatomical imaging modality. Such an anatomical-to-anatomical registration is usually easier to achieve than registration of fundamentally different functional and anatomical images.

The present paper first describes the process of data acquisition based upon this principle. Subsequent registration and image fusion of MRI and fMRI are presented next. Registration accuracy is assessed using an adapted set of images originating from the Vanderbilt retrospective registration project [3]. Finally, a patient case is presented, demonstrating the application of the described techniques to intraoperative navigation in neurosurgery.

\section{Materials and Methods}

\subsection{Image Acquisition}

MRI data originated from a 1.5T MRI scanner (Gyroscan ACS NT, Philips, Best, The Netherlands). Three different data sets are required:

(I) For fMRI computation, 5 slices of $\mathrm{T} 2 *$-weighted MRI were repeatedly collected using a fast gradient echo sequence. Imaging parameters were: pixel size $1.80 \mathrm{~mm} \times 1.80 \mathrm{~mm}(128 \times 128$ pixels $)$, slice thickness $7.0 \mathrm{~mm}$, no gaps. Functional MRI images were computed using evaluation software provided by Philips (Brain Activation Processing Tool, Release 6.1). Functional regions of interest were then identified in fMRI and transferred to one of the original $\mathrm{T} 2{ }^{*}$-weighted images (Figure 2). 


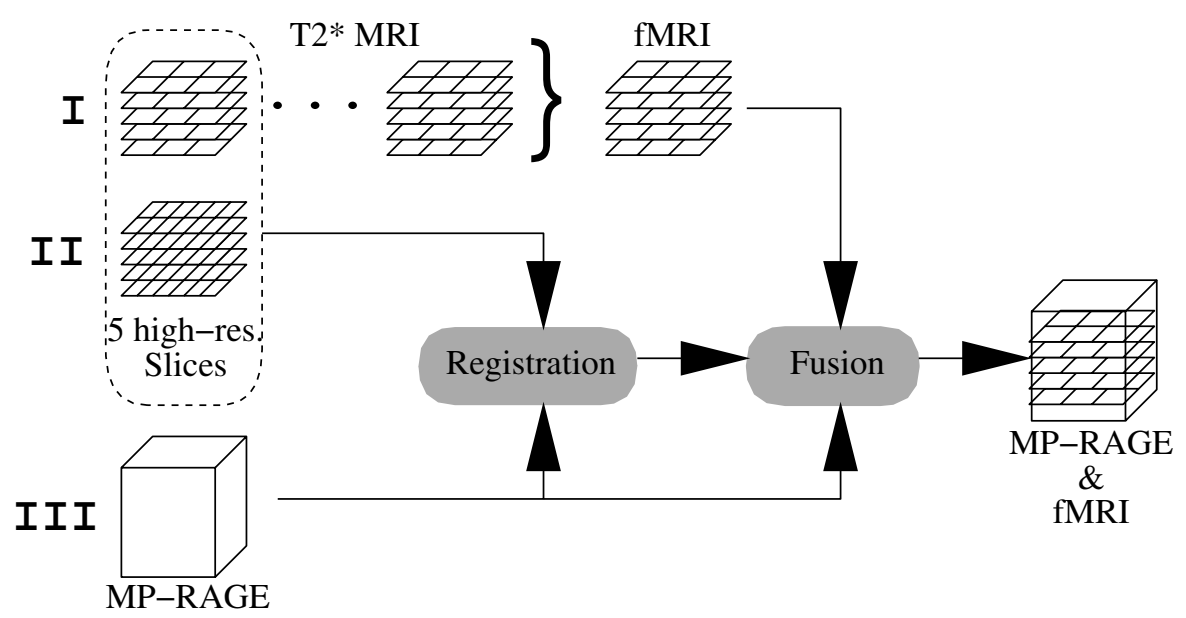

Fig. 1. Flow of image data through the registration and fusion process. A set of $5 \mathrm{~T} 2{ }^{*}$-weighted MRI images $(I)$ is acquired repeatedly. From this sequence, fMRI images are computed. In addition, 5 high-resolution anatomical images $(I I)$ are collected at the same locations. These are registered to an MP-RAGE volume $(I I I)$. For fusion with the MP-RAGE data, the resulting transformation can be applied to fMRI as well, because the spatial locations of fMRI slices are identical to those of the $\mathrm{T} 2{ }^{*}$-weighted slices as well as the additional anatomical slices.

(II) For registration an additional set of 5 anatomical slices was collected. These were measured immediately after the $\mathrm{T} 2{ }^{*}$-weighted series and at the very same spatial locations (Figure 2). Parameters for these slices were: pixel size $0.45 \mathrm{~mm} \times 0.45 \mathrm{~mm}(512 \times 512$ pixels $)$, slice thickness $7.0 \mathrm{~mm}$, no gaps.

(III) For navigation, high-resolution MRI data was collected using an MPRAGE sequence (called 3D-FFE on Philips scanners). Typical acquisition parameters were: pixel size $0.45 \mathrm{~mm} \times 0.45 \mathrm{~mm}(512 \times 512$ pixels $), 160$ slices, slice thickness $1.0 \mathrm{~mm}$, no gaps.

\subsection{Image Processing}

As mentioned above, fMRI images were computed from a series of $\mathrm{T} 2{ }^{*}$-weighted MRI volumes with a set of equivalent high-resolution slices measured immediately afterwards. For this reason, there is a trivial spatial relationship of functional and anatomical images (Figure 2). This is exploited for registration of fMRI to other anatomical imaging modalities as well. In the present paper for instance, this is a full MP-RAGE volume. Figure 1 illustrates the process.

Registration was performed by optimization of the normalized mutual information similarity measure [4]. The optimum rigid-body transformation was determined by an independent implementation of a multiresolution search algorithm similar to that described in [5]. Afterwards, MP-RAGE and fMRI were 


\begin{tabular}{|c|c|c|c|c|c|c|c|c|c|}
\hline Patient ID & 101 & 102 & 104 & 105 & 106 & 107 & 108 & 109 & \\
\hline & & $28-36$ & $\overline{24-32}$ & $24-32$ & $32-40$ & $16-24$ & $24-3$ & $24-32$ & \\
\hline Offset & 81 & 81 & 69 & 69 & 93 & 45 & 69 & 69 & {$[\mathrm{~mm}$} \\
\hline
\end{tabular}

Table 1. Selected slices and $z$-translation offsets for the patients from the Vanderbilt data used for registration accuracy assessment. Every other slice in the range given for each patient was contained in the sparse T2-weighted image (indexing starts with slice \#1). To compensate for this modification, the offsets in the second table row were added to the $z$-translation component of the resulting registration transformations. There original number of slices was 52 for all patients. Patient 103 is not included because no T2-weighted data was available for this patient.

fused by an overlay-based technique previously applied to CT and MRI [6,7]. The resulting images showed the ROIs defined in fMRI and the unaltered MP-RAGE data where no ROI was present (Figure 4).

\subsection{Validation of Registration Accuracy}

Accuracy of image registration was assessed using data originating from the Vanderbilt retrospective registration project [3]. As gold-standard transformations based on bone-implanted fiducial markers [8] are known for that data, the accuracy of the automatic registration algorithm could be quantified. From the data provided by that project, T2-weighted MRI to MP-RAGE registration was chosen to represent the situation of the present study. T2-weighted images were used, because they best matched the properties of the images used for fMRI computation.

From fMRI acquisition as described in section 2.1, only five anatomical MRI slices corresponding to fMRI were available for registration. To reflect this in the registration accuracy assessment, the original T2-weighted volumes of the Vanderbilt data (52 slices) were reduced accordingly. In particular, a subset of only five images with a uniform spacing of $6 \mathrm{~mm}$ (twice the original spacing) was selected from the original T2-weighted data. This was done to match the $7 \mathrm{~mm}$ spacing of fMRI data as closely as possible without having to apply inter-slice interpolation. Matrix size and pixel calibration of the original images remained unmodified.

In order to focus on regions of diagnostic interest, the range of remaining slices was chosen in such a way as to cover lesions identified independently for each of the patients. Table 1 gives the details about which slices were used from the T2-weighted data of the respective patients. It also contains information about how the resulting transformations had to be processed to be comparable to the available gold-standard registrations. 


\begin{tabular}{lrllllllll} 
& Patient ID 101 & 102 & 104 & 105 & 107 & 108 & 109 & total & \\
\hline \multirow{3}{*}{ Complete } & mean 2.78 & 1.81 & 1.90 & 2.54 & 3.43 & 2.51 & 2.68 & 2.59 & {$[\mathrm{~mm}]$} \\
& median 2.81 & 1.84 & 1.93 & 2.55 & 3.41 & 2.43 & 2.74 & 2.67 & {$[\mathrm{~mm}]$} \\
& $\max 2.95$ & 1.92 & 2.55 & 2.77 & 4.13 & 3.33 & 3.37 & 4.13 & {$[\mathrm{~mm}]$} \\
\multirow{3}{*}{ Sparse } & mean 2.39 & 1.33 & 1.17 & 2.38 & 3.99 & 2.07 & 2.08 & 2.20 & {$[\mathrm{~mm}]$} \\
& median 2.44 & 1.29 & 1.18 & 2.40 & 4.05 & 2.11 & 2.11 & 2.16 & {$[\mathrm{~mm}]$} \\
& $\max 2.98$ & 1.79 & 1.39 & 2.51 & 4.51 & 2.45 & 2.46 & 4.51 & {$[\mathrm{~mm}]$} \\
\hline
\end{tabular}

Table 2. Registration errors compared to the gold-standard for each patient and averaged over all patients (rightmost column). The upper three rows give the results for complete (52 slices, spacing $3 \mathrm{~mm}$ ) data. Below are the results for sparse (5 slices, spacing $6 \mathrm{~mm}$ ) data. Complying with the policy of the original Vanderbilt study, patient 106 was omitted as visual inspection revealed a failed registration.

\subsection{Surgical Navigation}

After fusion, the resulting images were reformatted to slice images spatially equivalent to the slices of the original MP-RAGE volume. These were written to DICOM files and subsequently communicated to an MKM operating microscope (Zeiss, Oberkochen, Germany) via campus-wide LAN. There, they were used for target definition as well as for intraoperative navigation (Figure 4).

\section{Results}

Registration Accuracy Assessment. The accuracy assessment results for registration of original and sparse Vanderbilt data are presented in Table 2. T2-weighted to MP-RAGE registration accuracy was not appreciably worse using only 5 slices. In fact, accuracy in terms of median errors was found to be even better in $6 / 7$ cases using sparse data than it was with the complete set of T2 slices considered.

Case Report. The procedure described above was applied to a patient suffering from a grade II-III (biopsy proved state III) cystic glioma. The anatomical and functional MRI images of this patient are shown in Figure 2. In Figure 3, a combined presentation of MP-RAGE and T2*-weighted MRI provides a visual impression of registration accuracy for this particular patient.

Fused data was also used for navigation during surgery. Figure 4 shows slices reformatted during surgery matching the current location in the operating region looked at by the surgeon. From the ROIs defined in fMRI and overlaid upon the anatomical MRI data, the spatial relationship of tumor, cyst, and Broca's area could clearly be recognized. By this presentation, the neurosurgeon performing the tumor resection was able to minimize damage to the patient's motor speech area. 

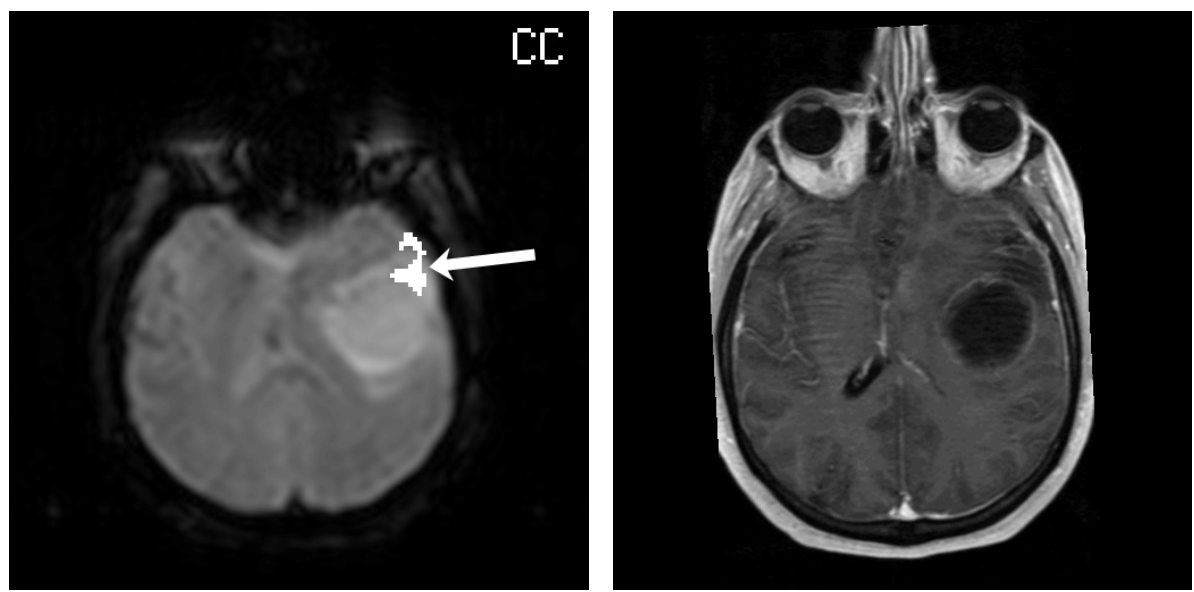

Fig. 2. Left: One of the T2*-weighted MRI images with overlaid functional information. The white area (arrow) adjacent to the cystic glioma represents Broca's area (motor speech center) identified in fMRI. Right: High-resolution anatomical slice measured at the same location.
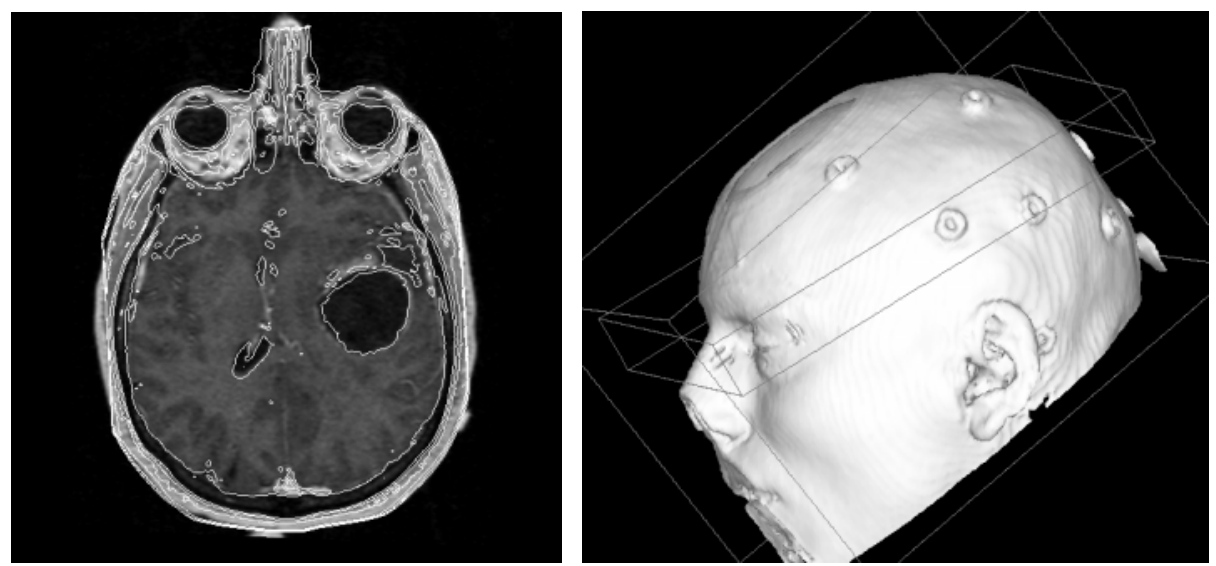

Fig. 3. Multi-modality images demonstrating registration accuracy of MPRAGE and fMRI. Left: MP-RAGE slice overlaid with isolines generated from the accordingly reformatted $\mathrm{T} 2 *$-weighted image used for fMRI computation. Right: spatial relationship of both modalities in 3D space. Bounding boxes represent the extents of MP-RAGE and fMRI volumes. The skin surface was computed by isosurface generation from the MP-RAGE data. On the back of the patient's head, the fiducials used for patient-to-data registration can be seen. 

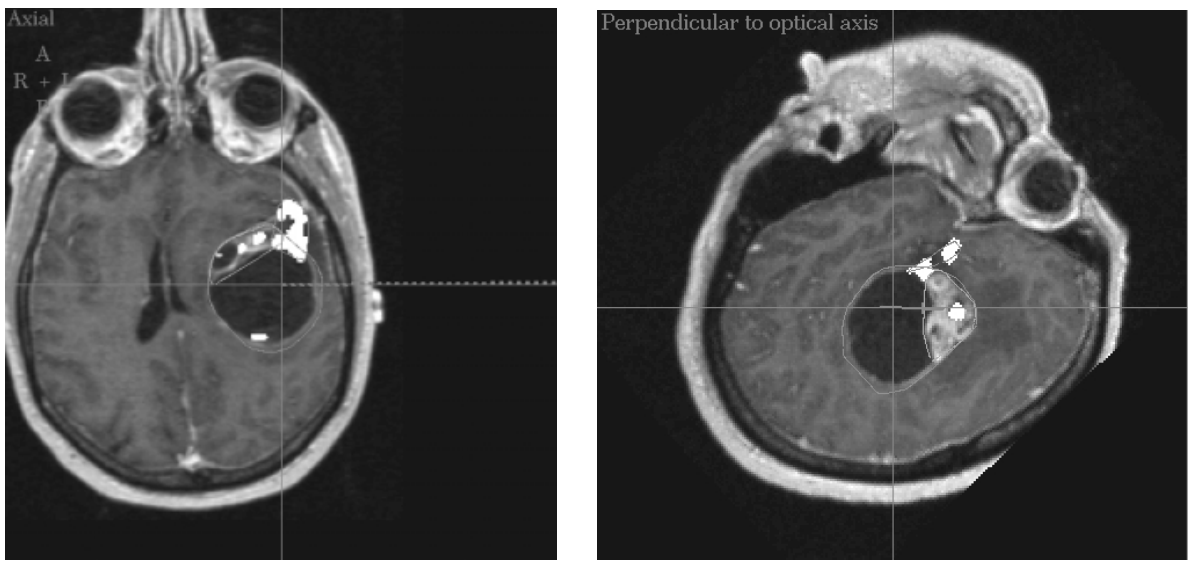

Fig. 4. Fused images corresponding to the operating region. During surgery, the axial image on the left was reformatted along the optical axis (dashed line) of the MKM operating microscope. Right: Corresponding image reformatted perpendicular to the optical axis is displayed. The cross in the right image represents the exact position the microscope was focused upon when the images were generated. In addition, during surgery planning contours outlining tumor and Broca's area had been defined. These were also overlaid on the microscope view presented to the surgeon for orientation during the intervention.

\section{Discussion}

A major advantage of fMRI is its inherent support for acquisition of spatially equivalent morphological images. Automated image registration with voxelbased techniques could therefore be performed with high reliability and accuracy.

Using the Vanderbilt data, accuracies found for sparse ( 5 slices) T2-weighted data were similar to those achieved using the complete set (52 slices) of images. Therefore, the small number of slices available did not impose a severe restriction to accuracy and reliability of the registration (cf. Table 2). The only failed registration (patient 106) could easily be identified by visual inspection. For real patients, this could be compensated for by repeating registration after manual determination of an initial registration estimate.

Two additional facts likely to have caused a decrease in registration accuracy using the Vanderbilt data should also be mentioned. First, in the application described the imaging properties of both data sets (T1-weighted and MP-RAGE MRI) are more similar to each other than the respective Vanderbilt images.

Second, registration accuracy was evaluated for a set of volumes of interest (VOIs) defined in the original images (cf. [3]). A large number of these VOIs, however, was no longer present in the sparse data. Therefore, VOIs neither available for registration, nor contributing to the resulting fusionated images may have caused accuracy to appear worse than it actually was. 


\section{Conclusions}

This paper has demonstrated that the close connection of anatomical and functional MRI imaging can be exploited for easy registration and fusion of both. By comparison to gold-standard transformations in a similar context (Vanderbilt study), registrations using a limited number of slices did not decrease registration accuracy.

Integration of image acquisition, processing, and surgical navigation into a common schedule allowed routine application of the techniques presented. Therefore, usage of functional MRI provides a promising method of gaining insight into relation of anatomical and functional brain structures during navigated neurosurgery.

\section{Acknowledgements}

This work was funded by Deutsche Forschungsgemeinschaft (DFG), Graduate School 331. For their generous support in doing this research, the authors would like to thank Prof. Dr. Dr. R. Felix, MD (Director of Department of Radiology, Virchow-Hospital), Prof. Dr. W. Lanksch (Director of Department of Neurosurgery, Virchow-Hospital), and Prof. Dr. N. Hosten, MD (Scientific coordinator of Graduate School 331). The images and the standard transformations for registration accuracy assessment were provided as part of the project, "Evaluation of Retrospective Image Registration", National Institutes of Health, Project Number 1 R01 NS33926-02, Principal Investigator, J. Michael Fitzpatrick, Vanderbilt University, Nashville, TN.

\section{References}

1. J. Frahm, H. Bruhn, K. D. Merboldt, and W. Hanicke. Dynamic MR imaging of human brain oxygenation during rest and photic stimulation. J Magn Reson Imaging, 2(5):501-505, September 1992. 946

2. U.-W. Thomale, T. Liebig, C. Taschner, T. Rohlfing, J. Beier, A. Rosenthal, et al. Integration of functional MRI data in computer assisted surgery. In H. U. Lemke et al., editors, Computer Assisted Radiology and Surgery. Elsevier, June 1999. 946

3. J. West, J. M. Fitzpatrick, M. Y. Wang, B. M. Dawant, C. R. Maurer Jr, R. M. Kessler, et al. Comparison and Evaluation of Retrospective Intermodality Brain Image Registration Techniques. J Comput Assist Tomogr, 21(4):554-566, 1997. 946, 948, 951

4. C. Studholme, D. L. G. Hill, and D. J. Hawkes. An overlap invariant entropy measure of 3D medical image alignment. Pattern Recognition, 32:71-86, 1998. 947

5. C. Studholme, D. L .G. Hill, and D. J. Hawkes. Automated three-dimensional registration of magnetic resonance and positron emission tomography brain images by multiresolution optimization of voxel similarity measures. Med Phys, 24(1):2535, January 1997. 947 
6. D. L. Hill, D. J. Hawkes, J. E. Crossman, M. J. Gleeson, T. C. Cox, E. E. Bracey, et al. Registration of MR and CT images for skull base surgery using point-like anatomical features. Br J Radiol, 64(767):1030-1035, November 1991. 948

7. N. Hosten, T. Rohlfing, J. Beier, T. Liebig, W. Lanksch, and R. Felix. Registration of CT and MRT images for navigation in image-guided brain surgery. Radiology, 209 (P):206, November 1998. 948

8. C. R. Maurer, Jr, J. M. Fitzpatrick, M. Y. Wang, R. L. Galloway, Jr, R. J. Maciunas, and G. S. Allen. Registration of head volume images using implantable fiducial markers. IEEE Trans Med Imaging, 16(4):447-462, August 1997. 948 